\title{
ANALYSIS OF THE IMAGE QUALITY OF NO GROUND CONTROLLED POSITIONING PRECISION ABOUT SURVEYING AND MAPPING SATELLITE
}

\author{
Wang $\mathrm{Yi}^{1 *}, \mathrm{Hu} \mathrm{Xin}^{2}$, Yang Xiafang ${ }^{3}, \mathrm{Xie}$ Guojun ${ }^{3}$ \\ ${ }^{1 .}$ Chang'an University, 710054 Xi'an, China - wangyi@geovis.com.cn \\ 2. Xi 'an institute of surveying and mapping, 710054 Xi'an, China - Huxin327@163.com \\ 3. Xi'an Zhong Ke Xing Tu Space Date Technology Co., Ltd, 710100 Xi'an, China - Yangxf@ geovis.com.cn
}

Commission III, ICWG III/IVb

KEY WORDS: Positioning accuracy, Modulation Transfer Function, Signal to Noise Ratio, Quantization Bits, degradation data

\begin{abstract}
:
The image quality of the surveying camera will affect the stereoscopic positioning accuracy of the remote sensing satellite. The key factors closely related to the image quality are Modulation Transfer Function(MTF),Signal to Noise Ratio(SNR) and Quantization Bits(QB). In " Mapping Satellite-1" image as the background, research the effect of positioning precision about the image quality in no ground controlled conditions, and evaluate the quantitative relationship with the positioning precision. At last verify the validity of the experimental results by simulating three factors of the degraded data on orbit, and counting the number of matching points, the mismatch rate, and the matching residuals of the degraded data. The reason for the variety of the positioning precision was analyzed.
\end{abstract}

\section{INTRDUCTION}

The "Mapping Satellite-1" surveying camera takes stereoscopic images from the front, the forward and the back angles and achieves the precise positioning of the target through high-precision pose track data processing, stereo measurement and adjustment techniques( Wang et al.,2010; Hu et al.,2008; Wang et al.,2004). The main factors that affecting the positioning accuracy without control points are satellite attitude ,satellite position, camera distortion parameters, camera focal length and image matching accuracy( Wang et al.,2010; Tao et al.,2006; Wang et al.,2009). The first four factors can be corrected with on-orbit geometric calibration method and the positioning accuracy will be also improved. In theory, the image matching accuracy can be 1/100 pixel( Wang,1979), but it is far from the theoretical value for the influence by blurring, noise, gray deviation and other factors in practical application. Image quality is closely related to the matching accuracy, but it is lack of relevant quantitative results at home and aboard. In general, Modulation Transfer Function (MTF), signal-noise ratio (SNR), and quantization bits (QB) are often used to estimate the quality of remote sensing images. Their reduction will reduce the image matching accuracy, and influences the positioning accuracy in further( $\mathrm{Fu}$, et al.,2011). In this paper, the degraded images are obtained by adding MTF, SNR and QB to the "Mapping Satellite-1" images with related degraded model. Finally, the influence of image quality on positioning accuracy is analyzed without ground control points.

\section{METHODOLOGY}

\subsection{The theory of positioning without ground control}

* Corresponding author: Wang Yi - wangyi@ geovis.com.cn

Foundation support: Chinese National Science Foundation (4157012140)hk

\section{points and making the degraded data}

The "Mapping satellite-1" eliminates system error of star sensor and GPS device with on-orbit calibration technology, and then improves the positioning accuracy in no ground controlled points by eliminating the accident error of exterior orientation elements with triangulation method. The specific idea is shown in Figure 1.

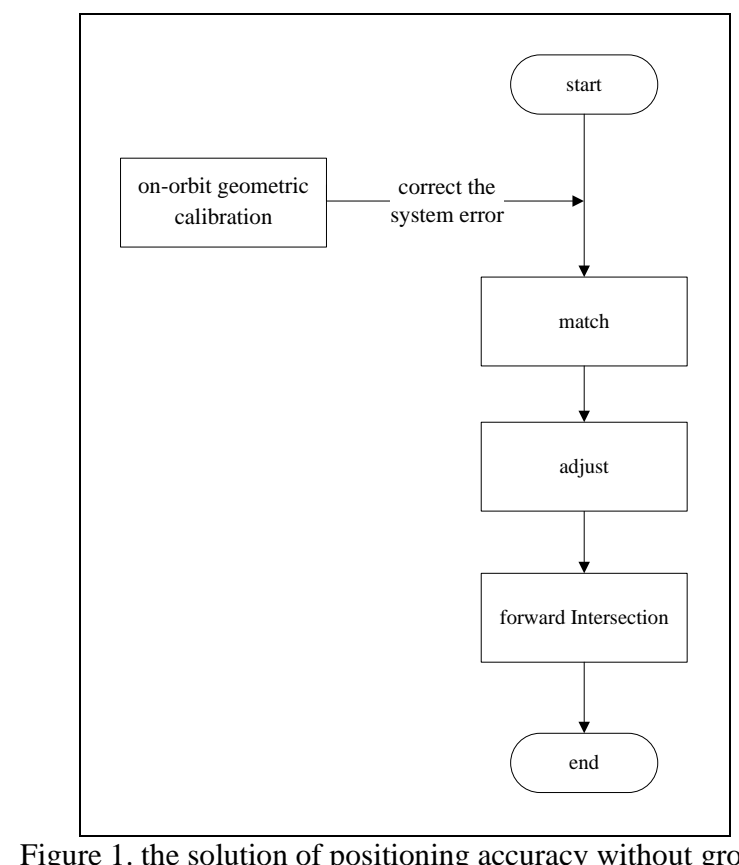

Figure 1. the solution of positioning accuracy without ground control points

A series of degraded data is obtained with MTF, SNR and QB processing on the "Mapping Satellite-1" images. The uncontrolled positioning accuracy is calculated from the series 
of degraded data. The influence of image quality on positioning accuracy is analyzed without ground control points.

The MTF degradation data is obtained by convoluting the three-line-array images with different gaussian blurring kernels as follows:

$$
I_{M T F}=I * G
$$

Where $\quad \boldsymbol{I}_{M T F}$ denotes the MTF degraded image

$I$ denotes the original image

$\boldsymbol{G}$ denotes the gaussian blurring kernel

Gaussian blurring kernel is as follows:

$$
G(x, y)=\frac{1}{2 \pi \sigma^{2}} \exp \left(-\left((x-m / 2)^{2}+(y-n / 2)^{2} / 2 \sigma^{2}\right)\right.
$$

Where $\quad \sigma$ denotes the standard deviation

The bigger it is, more blurry the image is

Different white gaussian noise is added to the three-line-array images to get the SNR degraded data as follows:

$$
I_{S N R}=I+N
$$

Where $\quad \boldsymbol{I}_{S N R}$ denotes the SNR degraded image

$I$ denotes the original image and

$\boldsymbol{N}$ denotes the white Gaussian noise with variance

The bigger $\sigma$ is, more blurry the image is.

The QB degraded data is obtained by stretching the bits of

\begin{tabular}{|c|c|c|c|c|c|c|c|c|c|}
\hline \multirow[b]{2}{*}{ MTF } & \multicolumn{3}{|c|}{ Image 1} & \multicolumn{3}{|c|}{ Image 2} & \multicolumn{3}{|c|}{ Image 3} \\
\hline & $\begin{array}{l}\text { plane } \\
(\mathrm{m})\end{array}$ & $\begin{array}{c}\text { elevation } \\
\text { (m) }\end{array}$ & $\begin{array}{c}\text { matching } \\
\text { points }\end{array}$ & $\begin{array}{c}\text { plane } \\
(\mathrm{m})\end{array}$ & $\begin{array}{c}\text { elevation } \\
\text { (m) }\end{array}$ & $\begin{array}{c}\text { matching } \\
\text { points }\end{array}$ & $\begin{array}{l}\text { plane } \\
(\mathrm{m})\end{array}$ & $\begin{array}{c}\text { elevation } \\
\text { (m) }\end{array}$ & $\begin{array}{c}\text { matching } \\
\text { points }\end{array}$ \\
\hline 0.001 & 54.1 & 33.5 & 17 & 33.7 & 31.9 & 10 & 22.0 & 16.3 & 21 \\
\hline 0.015 & 14.5 & 12.3 & 31 & 13.7 & 11.5 & 39 & 11.6 & 13.5 & 33 \\
\hline 0.028 & 11.3 & 6.6 & 198 & 11.0 & 6.6 & 186 & 11.1 & 6.5 & 203 \\
\hline 0.044 & 11.2 & 6.6 & 200 & 11.1 & 6.4 & 186 & 11.1 & 6.5 & 204 \\
\hline 0.066 & 11.3 & 6.5 & 197 & 11.1 & 6.5 & 188 & 11.0 & 6.5 & 204 \\
\hline 0.095 & 11.2 & 6.5 & 199 & 11.0 & 6.5 & 189 & 11.0 & 6.6 & 204 \\
\hline 0.131 & 11.2 & 6.5 & 199 & 11.0 & 6.6 & 176 & 11.0 & 6.6 & 203 \\
\hline 0.172 & 11.2 & 6.6 & 196 & 11.1 & 6.6 & 184 & 10.8 & 6.6 & 203 \\
\hline 0.217 & 11.2 & 6.6 & 196 & 11.2 & 6.5 & 181 & 10.8 & 6.7 & 203 \\
\hline 0.257 & 11.2 & 6.5 & 193 & 11.1 & 6.4 & 183 & 10.8 & 6.7 & 204 \\
\hline
\end{tabular}
three-line-array images linearly.

\subsection{Computing the positioning accuracy without control}

Table 1 . The plane error, elevation error and number of matching points with different MTF

\subsection{SNR experiment}

$$
S N R=10 \log _{10}\left(\delta_{\max }^{2} / \delta_{\min }^{2}\right)
$$

SNR and positioning accuracy are computed from SNR degradation data, and the SNR ranges from $10 \mathrm{~dB}$ to $60 \mathrm{~dB}$. The SNR is computed as follows: points

SIFT method is used to select the seed points, matching points are obtained with the methods of least square and gray removing the gross error points with the methods of biconditional restriction and random sample consensus.

A rigorous geometric model is established with the constraint relationship between the three-line-array images. The model is to adjust with the image connection points, solve the exact elements of outer orientation and fit the rational polynomial coefficient (RPC)

The positioning accuracy of check points is verified with the method of forward intersection.

controlled positioning accuracy is calculated from the degraded data, and the relationship between MTF, SNR, QB and positioning accuracy is analyzed.

\section{EXPERIMENTS AND RESULTS}

\subsection{MTF experiment}

MTF and positioning accuracy are computed from MTF degradation data, and the MTF ranges from 0.012 to 0.292 . The knife-edge method is used to calculate the MTF( Chen et al.,2009; Yang et al.,2005; Dennis et al.,2001; Leger et al.,2003; Forster et al.,1994). which is the value at the Nyquist frequency. We analyze the relationship between MTF and plane error, elevation error and matching points.

The results in Table 1 show that the plane error and elevation error change largely when the MTF value is less than 0.02 , and are hardly influenced when the MTF value is bigger than 0.02 . correlation, finally, the image connection points are obtained by 
We analyze the relationship between SNR and plane error, elevation error and matching points.

The results in Table 2 show that the plane error and elevation error are both large when SNR is less than 20db, and are hardly influenced when the SNR is larger than $20 \mathrm{db}$.

\begin{tabular}{|c|c|c|c|c|c|c|c|c|c|}
\hline \multirow{3}{*}{$\mathrm{SNR}(\mathrm{db})$} & \multicolumn{3}{|c|}{ Image 1} & \multicolumn{3}{|c|}{ Image 2} & \multicolumn{3}{|c|}{ Image 3} \\
\hline & plane & elevation & matching & plane & elevation & matching & plane & elevation & matching \\
\hline & (m) & $(\mathrm{m})$ & points & $(\mathrm{m})$ & $(\mathrm{m})$ & points & $(\mathrm{m})$ & $(\mathrm{m})$ & points \\
\hline 10 & \multirow{2}{*}{\multicolumn{3}{|c|}{ matching failure }} & \multicolumn{3}{|c|}{ matching failure } & \multirow{2}{*}{\multicolumn{3}{|c|}{ matching failure }} \\
\hline 15 & & & & 12.8 & 16.2 & 5 & & & \\
\hline 18 & 18.1 & 18.4 & 17 & 11.0 & 18.8 & 22 & 24.7 & 26.0 & 14 \\
\hline 20 & 11.1 & 6.8 & 42 & 11.1 & 6.5 & 56 & 11.4 & 6.0 & 30 \\
\hline 25 & 11.1 & 6.9 & 90 & 11.1 & 6.6 & 166 & 11.3 & 6.6 & 96 \\
\hline 30 & 11.1 & 6.7 & 133 & 11.0 & 6.7 & 197 & 11.4 & 6.8 & 126 \\
\hline 33 & 11.2 & 6.6 & 140 & 10.8 & 6.8 & 200 & 11.1 & 6.7 & 151 \\
\hline 36 & 11.1 & 6.8 & 147 & 11.0 & 6.5 & 201 & 11.2 & 6.7 & 156 \\
\hline 39 & 11.2 & 6.7 & 123 & 10.9 & 6.6 & 198 & 11.1 & 6.8 & 155 \\
\hline 42 & 11.1 & 6.8 & 157 & 10.7 & 6.8 & 199 & 11.3 & 6.8 & 153 \\
\hline 45 & 11.1 & 6.8 & 155 & 10.7 & 6.7 & 198 & 11.3 & 6.8 & 155 \\
\hline 48 & 11.2 & 6.9 & 149 & 10.7 & 6.8 & 197 & 11.2 & 6.7 & 156 \\
\hline
\end{tabular}

Table 2. the plane error, elevation error and number of matching points with different SNR

\subsection{QB experiment}

Positioning accuracy is computed from QB degradation data and the $\mathrm{QB}$ ranges from 3 to 15 .
The results in Table 3 show that it is fail to match when the QB is 3 or 4, the plane error and elevation error are large when the QB was 5 or 6 and are hardly influenced when the QB is larger than 6 .

\begin{tabular}{|c|c|c|c|c|c|c|c|c|c|}
\hline \multirow[b]{2}{*}{ QB } & \multicolumn{3}{|c|}{ Image 1} & \multicolumn{3}{|c|}{ Image 2} & \multicolumn{3}{|c|}{ Image 3} \\
\hline & $\begin{array}{l}\text { plane } \\
\text { (m) }\end{array}$ & $\begin{array}{l}\text { elevation } \\
\text { (m) }\end{array}$ & $\begin{array}{c}\text { matching } \\
\text { points }\end{array}$ & $\begin{array}{l}\text { plane } \\
(\mathrm{m})\end{array}$ & $\begin{array}{l}\text { elevation } \\
\text { (m) }\end{array}$ & $\begin{array}{c}\text { matching } \\
\text { points }\end{array}$ & $\begin{array}{l}\text { plane } \\
\text { (m) }\end{array}$ & $\begin{array}{c}\text { elevation } \\
\text { (m) }\end{array}$ & $\begin{array}{c}\text { matching } \\
\text { points }\end{array}$ \\
\hline 3,4 & \multicolumn{3}{|c|}{ matching failure } & \multicolumn{3}{|c|}{ matching failure } & \multicolumn{3}{|c|}{ matching failure } \\
\hline 5 & 64.1 & 34.5 & 5 & 51.4 & 32.6 & 5 & 112.1 & 78.2 & 5 \\
\hline 6 & 20.1 & 11.4 & 17 & 16.1 & 26.3 & 22 & 64.1 & 26.0 & 14 \\
\hline 7 & 11.4 & 6.3 & 156 & 11.4 & 6.0 & 153 & 10.8 & 7.0 & 174 \\
\hline 8 & 11.4 & 6.5 & 164 & 11.6 & 5.9 & 162 & 10.6 & 6.6 & 180 \\
\hline 9 & 11.2 & 6.5 & 172 & 11.7 & 5.9 & 167 & 10.6 & 6.6 & 180 \\
\hline 10 & 11.2 & 6.5 & 175 & 11.7 & 6.0 & 168 & 10.6 & 6.6 & 183 \\
\hline 11 & 11.2 & 6.6 & 175 & 11.7 & 6.0 & 168 & 10.6 & 6.6 & 182 \\
\hline 12 & 11.2 & 6.6 & 175 & 11.6 & 6.0 & 168 & 10.6 & 6.6 & 180 \\
\hline 13 & 11.2 & 6.6 & 175 & 11.7 & 6.0 & 168 & 10.6 & 6.6 & 182 \\
\hline 14 & 11.2 & 6.6 & 175 & 11.7 & 6.1 & 168 & 10.6 & 6.6 & 179 \\
\hline 15 & 11.2 & 6.6 & 175 & 11.6 & 5.9 & 168 & 10.6 & 6.6 & 181 \\
\hline
\end{tabular}

Table 3. the plane error, elevation error and number of matching points with different QB

\subsection{Analysis of experiment}

The experimental results demonstrate that only in the case of extremely poor image, the positioning accuracy will be serious worse. In other cases, despite the image quality is different, it will have little influence on the positioning accuracy. Whether such a conclusion is correct, an evidence will be given. The detailed results of the first simulated image are shown in Table 4.

There are three steps to calculate the positioning accuracy, which are automatic matching, iterative adjustment and 
eliminating mismatch points. The positioning accuracy is the result of adjustment. Table 4 gives the mismatch rate, matching residual and matching residual after mismatching points are eliminated. The definitions in the table are as follows:

(1) Mismatch rate

$$
M R=\frac{N E M P}{N M P} \times 100 \%
$$

Where $\quad M R$ denotes mismatch rate

$N E M P$ denotes the number of mismatch points

$N M P$ denotes the number of matching points

The number of matching points is the sum of correct matching points and mismatch points.

(2) Matching residual
The matching residual is the image square residual after the adjustment on automatic matching. The residual of single point is calculated as follows:

$$
\Delta x=x-x^{\prime} ; \Delta y=y-y^{\prime}
$$

Where $\quad(x, y)$ is the point coordinate before adjustment $\left(x^{\prime}, y^{\prime}\right)$ is the point coordinate after adjustment.

The matching residual is as follows:

$$
\Delta=\sqrt{\sum_{i=1}^{n}\left[\left(\Delta x_{i}\right)^{2}+\left(\Delta y_{i}\right)^{2}\right] / n}
$$

\begin{tabular}{|c|c|c|c|c|}
\hline MTF & mismatch rate & matching & residual (pixels) & $\begin{array}{l}\text { matching residual without mismatch points } \\
\qquad \text { (pixels) }\end{array}$ \\
\hline 0.0121 & $83.85 \%$ & & 36.07 & 24.25 \\
\hline 0.019 & $62.98 \%$ & & 14.23 & 13.16 \\
\hline 0.0282 & $26.85 \%$ & & 10.93 & 0.40 \\
\hline 0.0443 & $15.79 \%$ & & 8.89 & 0.40 \\
\hline 0.0666 & $18.06 \%$ & & 9.83 & 0.37 \\
\hline 0.0955 & $22.58 \%$ & & 8.11 & 0.39 \\
\hline 0.1313 & $26.34 \%$ & & 11.51 & 0.36 \\
\hline 0.1729 & $14.89 \%$ & & 7.33 & 0.36 \\
\hline 0.2178 & $22.22 \%$ & & 10.23 & 0.34 \\
\hline 0.2575 & $17.61 \%$ & & 7.92 & 0.35 \\
\hline 0.2614 & $15.76 \%$ & & 5.90 & 0.34 \\
\hline 0.293 & $27.07 \%$ & & 11.39 & 0.30 \\
\hline
\end{tabular}

Where $\quad n$ denotes the number of matching points.

Table 4. the mismatch rate, matching residual and matching residual without mismatch points with different MTF of the first simulated image

(3) The method of eliminating mismatch points

The method of triple mean square error is used to eliminate mismatch points: 1) calculate the mean square error of all of matching points denoted by $\sigma, 2$ ) eliminate the point which matching residual is bigger than $3 \sigma$.

The results in Table 4 show that the mismatch rate is kept within $30 \%$ and there is no obvious change with the change of image quality when the MTF value is not less than 0.0282 , the mismatch rate rises sharply and reaches $62.98 \%$ and $83.85 \%$ when the MTF value is less than 0.0282 . The matching residual is the same as mismatch rates. It increases sharply and is much larger than 1 pixel when the MTF value is less than 0.0282. The matching residual after the mismatch points are eliminated is obviously reduced, which is less than 1 pixel when the MTF value is not less than 0.0282 and still larger when the MTF value is less than 0.0282 . The reason is that the image quality is too bad and there are too many mismatch points that the mismatch points can't be effectively eliminated when the MTF value is less than 0.0282 . This leads to serious error inputs to later adjustment and then affects the positioning accuracy.
In a word, with the decline of image quality, the mismatch will occur. However, if the image quality is still not too bad, there is no obvious relationship between the mismatch and the image quality, and the positioning accuracy will not be influenced. The reason is that the mismatch points will be eliminated during the subsequent adjustment. Only in the case of extremely poor images, the mismatch rate is so large that the mismatch points cannot be eliminated and the positioning accuracy will be serious worse.

\section{CONCLUSION}

This paper analyses the influence factors on positioning accuracy of surveying and mapping. The degraded models of MTF, SNR and QB are established on three groups of on-orbit simulated images. The study focuses on the influence of the three factors on the positioning accuracy. It is concluded that the plane error and elevation error are large when the MTF is less than 0.02 and SNR is less than 20db and the QB is less than 6 , the plane error and elevation error will be ideal and remain stable when the MTF is bigger than 0.02 and the SNR is 
bigger than $20 \mathrm{db}$ and the QB is bigger than 6. This conclusion has clarified the specific influence of image quality (MTF, SNR, QB) on the positioning accuracy, and plays an important role in guiding the subsequent uncontrolled positioning of surveying and mapping satellite.

\section{REFERENCES}

Chen S.P, 2009. Some Issues about the remote sensing image quality. Spacecraft Recovery \& Remote Sensing, 30(2), pp. 46-51.

Dennis L. Helder, Taeyoung Choi, 2002. IKONOS Satellite in Orbit Modulation Transfer Function (MTF) Measurement, pp. 87-89.

Forster B C, Best P, 1994. Estimation of SPOT P-mode Point Spread Function and Derivation of a Deconvolution Filter. ISPRS Journal for RS and GIS, 49(6), pp. 32-42.

Fu P, Wang K, Meng J.B, et al, 2011. Research on influence of MTF on elevation precision in stereoscopic surveying. Spacecraft Recovery \& Remote Sensing, 32(6), pp. 68-73.

Hu X, Cao X.B, 2008. Analysis on precision of stereo mapping microsatellite using three-line array $\mathrm{CCD}$ images. Journal of Harbin Institute of Technology, 40(5), pp. 695-699.

Leger D, Viallefont F, Hillairet E, et al. 2003. In-flight
Refocusing and Refocusing and MTF Assessment of SPOTS. HRG and HRS.SPIE, 4881, pp. 224-231.

Tao J.S, Wang S.T, 2006. Analysis on large-scale space three-linear array camera precision sensitive factors. Optical Technique, 32(1), pp. 89-91.

Wang J.R, Wang R.X, Hu X, et al, 2009. The precision estimation of forward intersection of three-line array CCD imagery. Science of Surveying and Mapping, 34(4), pp. 9-11.

Wang R.X, Hu X, 2004. The difficult problem about satellite photogrammetry without control points. Science of Surveying and Mapping, 29(3), pp. 3-5.

Wang Z, Zhang L.P, Li Z.H, 2010. Imaging and error model of three-linear tridimensional mapping camera. Opto-Electronic Engineering, 37(1), pp. 95-100.

Wang Z, Zhang L.P, Yao H, 2010. Design on optical lens of three-linear tridimensional mapping camera. ACTA PHOTONICA SINICA, 39(2), pp. 228-231.

Wang Z.Z, 1979. Photogrammetric principle, Beijing: Press of Surveying and Mapping, pp. 32-78.

Yang B.X, 2005. Study on the SNR of TDICCD camera. Spacecraft Recovery \& Remote Sensing, 26(2), pp. 22-24. 